\title{
Electrostatically Tuning the Photodissociation of the Irgacure 2959 Photoinitiator in the Gas Phase by Cation Binding
}

\author{
Samuel J. P. Marlton ${ }^{\mathrm{a}}$, Benjamin I. McKinnon ${ }^{\mathrm{a}}$ \\ Nicholas S. Hill ${ }^{\mathrm{b}}$, Michelle L. Coote ${ }^{\mathrm{b}}$, and Adam J. Trevitt ${ }^{\mathrm{a}}$.
}

${ }^{a}$ Molecular Horizons and School of Chemistry and Molecular Bioscience, University of Wollongong, Wollongong, NSW 2522, AUSTRALIA,

${ }^{\mathrm{b}} \mathrm{ARC}$ Centre of Excellence for Electromaterials Science, Research School of Chemistry, Australian National University, Canberra, ACT 2601, AUSTRALIA

*Author to whom correspondence should be addressed: E-mail:

adamt@uow.edu.au (Adam J. Trevitt)

\begin{abstract}
The low-lying electronic states of Irgacure 2959, a Norrish-type I photoinitiator, complexed with a single metal cation are investigated in the gas-phase by photodissociation action spectroscopy. Analysis of the band-shifts using quantum chemical calculations (TD-DFT and SCS-CC2) reveals the underlying influence of the charge on the key electronic energy levels. Since the cations $\left(\mathrm{H}^{+}, \mathrm{Li}^{+}, \mathrm{Na}^{+}, \mathrm{K}^{+}, \mathrm{Zn}^{2+}, \mathrm{Ca}^{2+}\right.$ and $\left.\mathrm{Mg}^{2+}\right)$ bind at varying distances, the magnitude of the electric field at the center of the chromophore due to the cation is altered and this shifts the electronic states by different amounts. Photodissociation action spectra of cation-Irg complexes show that absorption transitions to the first ${ }^{1} \pi \pi^{*}$ state are red shifted with a magnitude proportional to the electric field strength (with red shifts $>1 \mathrm{eV}$ ) and in most cases the cation is essentially acting as a point-charge. Calculations show that a neighboring ${ }^{3} n \pi^{*}$ state, a key state for the $\alpha$-cleavage pathway, is destabilized (blue shifted) by the orientated electric field. As such, if the ${ }^{1} \pi \pi^{*-3} n \pi^{*}$ energy gap is reduced, increased inter-system crossing rates are expected resulting in higher yields of the desired radical photoproducts and this is controlled by the orientated electric field arising from the cation.
\end{abstract}

\section{Introduction:}

Theoretical and experimental investigations - particularly in combination - are providing new insights into the control of chemical reactivity using oriented electric field (OEFs). ${ }^{1-5}$ Recent experiments have shown OEFs can enhance ground state reactivity in a variety of contexts including homogenous catalysts in solution, ${ }^{6}$ single-molecules at junctions ${ }^{7}$ and charged groups interacting with radicals in the gas phase. ${ }^{8}$
Recently, Coote and co-workers used computational predictions to rationalize how OEFs can be used to tune the photodissociation properties of radical photoinitiators by shifting the key excited electronic quantum levels implicated in photodissociation. $^{9,} 10$ This requires the coordinated shifting of the absorption transition with the other states implicated in the dissociation to the desired products. 
Following absorption of a photon, it is often the interplay between the spectroscopically prepared excited state and the neighboring "dark" excited state(s) that mediate the photodissociation efficiency. ${ }^{9-12}$ As a result, the photon energy corresponding to the maximum absorption cross-section does not necessarily correspond to the maximum photodissociation efficiency. ${ }^{12-14}$ As red-shifting the active wavelengths of photoinitiators is often sought after, since lower energy photons are generally less expensive and are less damaging to other colocated molecules, it is vital that both the absorption transition and the other key states shift in accord to maintain high photodissociation yields. Predicting the impact of chemical modification, or application of OEFs, on photoactivity is not straightforward as different excited states have different polarities and polarizabilities and thus respond differently. ${ }^{9,10,15}$ This in turn has complex follow-on effects on intersystem crossing rates, triplet lifetimes and other factors affecting photodissociation. To study these processes, experiments that probe both excitation and dissociation as a function of photon energy are required and gas-phase photodissociation action spectroscopycombining laser photodissociation with $\mathrm{m} / z$-selected ions-is well suited to investigate the fundamental processes of photoinitiator systems.

Gas-phase photodissociation (PD) action spectroscopy provides information on the structural characterization of ions including the position of metal cations complexed to chromophores $^{16-22}$ and the location of protonation sites. ${ }^{23-31} \mathrm{PD}$ action spectroscopy of ions complements other experimental strategies examining the effect of electric fields on molecular reactivity. ${ }^{8,32-38}$

This paper focuses on the photodissociation of Irgacure 2959 (Irg), 2-hydroxy-4-(2-hydroxyethoxy)-2methylpropiophenone, a well-known photoinitiator based on acetophenone with Norrish type-I photo-chemistry ${ }^{9}, 10$ and popular for use in bio-printing. ${ }^{39-41}$ Irg absorbs in the UV region with an absorption maximum at $\lambda=273 \mathrm{~nm} .{ }^{42}$ Following absorption, the desired $\alpha$-cleavage photodissociation mechanism of Irg is summarized as a ${ }^{1} \pi \pi^{*} \rightarrow{ }^{1} \mathrm{n} \pi^{*} \rightarrow{ }^{3} \pi \pi^{*} \rightarrow{ }^{3} \mathrm{n} \pi^{*}$ $\rightarrow \alpha$-cleavage. ${ }^{9-11,43}$ Because neutral Irg has an $\mathrm{S}_{1}$ state with ${ }^{1} \mathrm{n} \pi^{*}$ character, the $\mathrm{S}_{1}\left({ }^{1} \mathrm{n} \pi^{*}\right) \rightarrow \mathrm{T}_{\mathrm{n}}\left({ }^{3} \pi \pi^{*}\right)$ ISC pathway-the second step-should be favorable (El Sayed Rules). ${ }^{44}$ Ultimately, dissociation from the ${ }^{3} \mathrm{n} \pi *$ state correlates to ${ }^{\circ} \mathrm{C}_{9} \mathrm{H}_{9} \mathrm{O}_{3}$ $+{ }^{\bullet} \mathrm{C}_{3} \mathrm{H}_{6} \mathrm{OH} \alpha$-hydroxyalkyl radical. ${ }^{43}$ The quantum yield of $\alpha$ cleavage from Irg is $\phi_{\alpha}=0.29$ in acetonitrile. ${ }^{42}$ Irg has a strong $\pi \rightarrow \pi^{*}$ transition $\left(\varepsilon=16200 \mathrm{M}^{-1} \mathrm{~cm}^{-1}\right.$ in acetonitrile) ${ }^{45}$ which is excited, for example, in the cross linking of hydrogels. ${ }^{46}$ Alternatively, to reduce damage in bio-applications, lower energy photons $(\lambda>320 \mathrm{~nm})$ can be used and this directly accesses the weaker $\mathrm{n} \rightarrow \pi^{*}$ transition $\left(\varepsilon=4 \mathrm{M}^{-1} \mathrm{~cm}^{-1}\right.$ for $\lambda=$ $365 \mathrm{~nm}$ in ethanol $)^{39-41,47}$ and thus requires longer irradiation times. ${ }^{46,48}$ This trade-off highlights the need to balance the efficiency of the absorbing transition, which often coincides with higher energy photons, with yields of $\alpha$-cleavage and other photo-initiated chemistry.

Hill et al. recently outlined that oriented electric fields can be used to (a) decrease the excitation energy of Irg and (b) increase the efficiency of $\alpha$-cleavage pathway. ${ }^{10}$ Their study targeted that fact that the transition dipoles for the ${ }^{1} \pi \pi^{*}$ and ${ }^{3} \mathrm{n} \pi^{*}$ states are anti-aligned (as depicted schematically in Figure 1) and thus are likely perturbed by OEFs in opposite directions. In addition, the $\pi \pi^{*}$ states are highly polarizable, which greatly enhances their stabilization $(>1 \mathrm{eV})$ in the presence of an appropriately aligned field. Thus, suitable modification with a charged group or metal cation can lower the ${ }^{1} \pi \pi^{*}$ state to become the $\mathrm{S}_{1}$ and then, in the absence of any lower lying ${ }^{1} n \pi^{*}$, the $\mathrm{S}_{1}\left({ }^{1} \pi \pi^{*}\right) \rightarrow \mathrm{T}_{\mathrm{n}}\left({ }^{3} \mathrm{n} \pi^{*}\right)$ ISC pathway can dominate and enhance $\alpha$-cleavage. ${ }^{9-11}$ The efficiency of this pathway will be influenced by the energy gap between the ${ }^{1} \pi \pi^{*}$ and ${ }^{3} \mathrm{n} \pi^{*}$ states and a smaller energy gap is expected to increase the ISC rate and increase $\alpha$-cleavage yields. ${ }^{9-11}$

Motivated by these predictions, the present study targets Irg bound to metal cations in the gas phase. Using PD action spectroscopy together with mass spectrometry allows for investigation of the energy shift of the ${ }^{1} \pi \pi^{*}$ states of Irgacure in the presence of different cations $\left(\mathrm{M}^{\mathrm{Q}}\right)$ along with detection of the photoproduct ions. It is observed that the energies of the electronic quantum states are shifted by the presence of the $\mathrm{M}^{\mathrm{Q}^{+}}$ions which altered the (a) the absorption profile and (b) subsequent photodissociation.

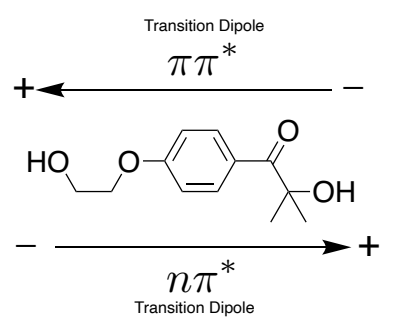

Figure 1. Alignment of transition dipoles for the $n \pi^{*}$ and $\pi \pi^{*}$ transitions for Irgacure as described by Hill et al. ${ }^{10}$

\section{Results and Discussion:}

\section{Structures:}

In order to characterize the effect of oriented electric fields (OEFs) arising from the presence of $\mathrm{M}^{\mathrm{Q}^{+}}$charged atoms on the electronic quantum states of Irg, it is first necessary to determine the preferred location of the charged atom. Based on the $m / z$ values of the ions, the $\mathrm{M}^{+}$cations gave rise to complexes of one Irg molecule and one $\mathrm{M}^{+}$, whereas the $\mathrm{M}^{2+}$ cations gave rise to complexes comprising two Irg molecules clustered one $\mathrm{M}^{2+}$, with essentially no reproducible signal detected for single Irg- $\mathrm{M}^{2+}$ clusters. Figure 2 shows structure of $\mathrm{M}^{+}\left(\mathrm{M}^{+}=\mathrm{H}^{+}, \mathrm{Li}^{+}\right.$, $\mathrm{Na}^{+}, \mathrm{K}^{+}$) ions complexed with one Irgacure 2959 molecule, which are labelled Irg- $\mathrm{M}^{\mathrm{Q}^{+}}$in general. Figure 2 also shows structures for $\mathrm{M}^{2+}=\mathrm{Zn}^{2+}, \mathrm{Mg}^{2+}, \mathrm{Ca}^{2+}$ clusters and these are labelled as Irg- $\mathrm{M}^{\mathrm{Q}+}$-Irg. The relative energies of other calculated Irg- $\mathrm{M}^{\mathrm{Q}^{+}}$structures are shown in Figure S2 in Supporting Information. In all cases, Structure $\mathrm{A}\left(\mathrm{M}^{\mathrm{Q}+}\right.$ binding to the ketone and hydroxide oxygens), as shown in Figure 2, is the lowest energy structure. 


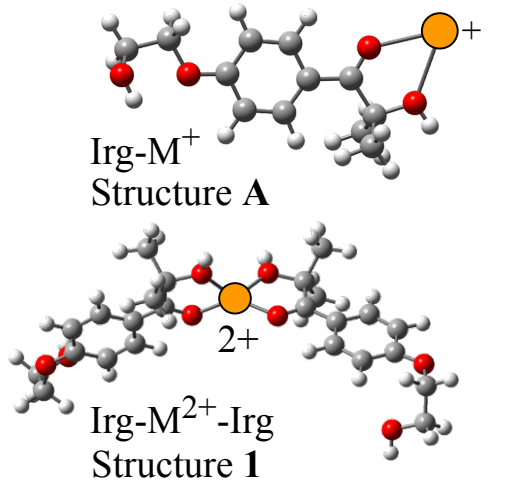

Figure 2. Lowest energy structures for Irg- $\mathrm{M}^{+}$and $\operatorname{Irg}-\mathrm{M}^{2+}-\mathrm{Irg}$ complexes as calculated using M06-2X.

It is known that thermodynamically less-favored gas-phase structures can survive ESI since they are kinetically trapped. ${ }^{49-}$ 53 However it is determined that structures other than the minimum-energy structure A can be excluded from the ensuing analysis based on DFT energies and based on their predicted electronic spectra residing above the $3.0 \mathrm{eV}$ to $5.0 \mathrm{eV}$ range. Also, structures could be excluded based on low isomerization barriers to form Structure A. For additional details relating to the structure assignment see Supporting Information Section SI.2.

The lowest energy structure of Irg- $\mathrm{M}^{2+}$-Irg (labelled Structure 1) has the hydroxy and ketone oxygens bound to the $\mathrm{M}^{\mathrm{Q}^{+}}$ ion. This Irg- $\mathrm{M}^{2+}$-Irg structure has some underlying $\mathrm{C}_{2}$-like symmetry for the chromophore but not for the flexible hydroxyethane tails. Based on the relative energies of Irg- $\mathrm{M}^{2+}$ Irg structures as calculated with M06-2X (Figure S5 of Supporting Information), the following analysis assumes the results are related to Structure 1.

\section{Action Spectroscopy}

Figure 3 contains the photodissociation spectra (black line) of Irg- $\mathrm{M}^{+}$and Irg- $\mathrm{M}^{2+}$-Irg ions alongside SCS-CC2 calculated vertical transitions below $5.0 \mathrm{eV}$ (green bars). The PD action spectra are plotted as the sum of all photoproduct intensities. Representative product mass spectra are shown Figure S6 of the Supporting Information. The $\mathrm{Na}^{+}$and $\mathrm{K}^{+}$complexes form $\alpha$-cleavage pathway products as a dominant photoproduct pathway, the $\mathrm{Li}^{+}$system shows small amounts and the $\mathrm{H}^{+}$ and the $\mathrm{M}^{2+}$ cases exhibit a range of photoproducts.

The lowest energy peaks in experimental action spectra are fitted with a Gaussian function (blue line in Figure 3), which is used to assign the position of maximum PD efficiency. Additional Gaussian functions are fitted to include the higher energy peaks and are shown in Figure S7 of Supporting Information. The center of this blue Gaussian fit (labelled $\mathrm{E}_{\text {exp }}$ ) will be used and compared with calculated vertical transition energies, although it is worth noting that experimental assignment of the vertical transition energy is subjective. ${ }^{23,54,55}$

The $E_{\text {exp }}$ values are listed in Table 1 along with TD-DFT and SCS-CC2 vertical transition energies $\left(\mathrm{E}_{\mathrm{vert}}\right)$ for Structure $\mathrm{A}$ of Irg- $\mathrm{M}^{+}$and Structure 1 of Irg- $\mathrm{M}^{2+}-\mathrm{Irg}$. The $\mathrm{E}_{\mathrm{vert}}$ values calculated using SCS-CC2 and TD-DFT generally agree with $\mathrm{E}_{\text {exp }}$, assuming the peak maximum corresponds to the vertical transition energy, but with a systematic overestimation of $c a$. $0.3 \mathrm{eV}$ for SCS-CC2 and $c a .0 .5 \mathrm{eV}$ for TD-DFT, which is consistent with other reports using these methods. ${ }^{23,27,54,56}$ Two features are observed for Irg- $\mathrm{M}^{\mathrm{Q}^{+}}$-Irg complexes in the PD action spectra and these are predicted by the SCS-CC2 calculations. Using these calculations, the experimental PD spectra are assigned to the $\pi \rightarrow \pi^{*}$ transitions of either Structure A for Irg- $\mathrm{M}^{\mathrm{Q}^{+}}$or Structure 1 for $\operatorname{Irg}-\mathrm{M}^{\mathrm{Q}^{+}}-\mathrm{Irg}$, for each cation.

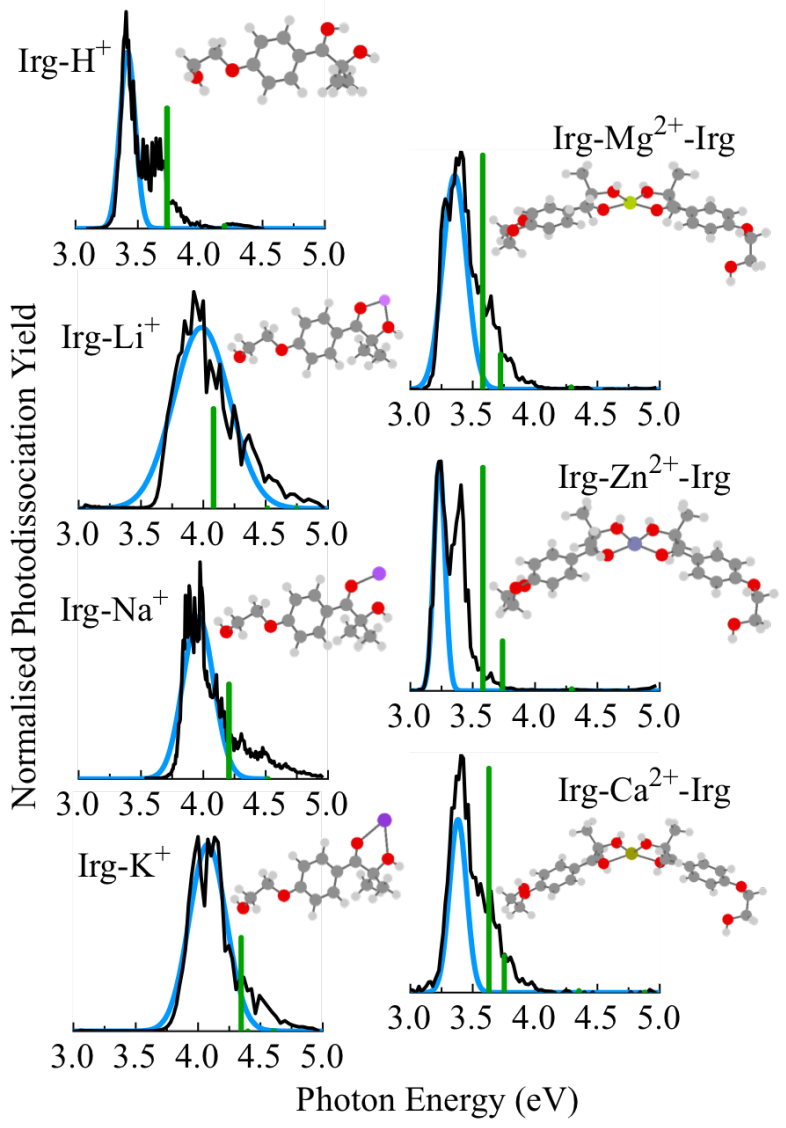

Figure 3. Photodissociation action spectra of Irgacure $2959 \mathrm{M}^{\mathrm{Q}^{+}}$ complexes $\left(\mathrm{M}^{\mathrm{Q}+}=\mathrm{H}^{+}, \mathrm{Li}^{+}, \mathrm{Na}^{+}, \mathrm{K}^{+}, \mathrm{Mg}^{2+}, \mathrm{Zn}^{2+}, \mathrm{Ca}^{2+}\right)$. Single Gaussian functions (blue) are fit to the lowest energy transition. Additional Gaussian functions used for fitting are shown in Figure S7 of Supporting Information. Green sticks are the calculated vertical transition energies $\left(E_{\text {vert }}\right)$ for each lowest energy structure using SCS-CC2 with bar heights equal to the oscillator strengths for each respective transition.

\section{${ }^{1} \pi \pi *$ State Shift by $\mathrm{M}^{+}$and $\mathrm{M}^{2+}$ cations.}

Neutral Irg has an $\mathrm{S}_{1}$ state with ${ }^{1} \mathrm{n} \pi^{*}$ character and an $\mathrm{S}_{2}$ state with ${ }^{1} \pi \pi^{*}$ character. Figure 4 shows the dominant contributing orbitals to these $S_{1}$ and $S_{2}$ transitions. The highest occupied $\pi$ and lowest unoccupied $\pi^{*}$ orbitals are primarily localized across the phenyl ring $\left(\mathrm{C}_{1-6}\right)$ in addition to on the ether oxygen and the ketone $\mathrm{C}_{7} \mathrm{O}_{1}$ groups (for atom labelling see Figure $\mathrm{S} 8(\mathrm{~A})$ ) and these groups comprise the chromophore of Irg. The ${ }^{1} \pi \pi^{*}$ transition shown in Figure 4 involves a redistribution of the electron density around aromatic ring as well as an increase around the ketone moiety. In contrast, the ${ }^{1} \mathrm{n} \pi^{*}$ transition shifts electron density from the $\mathrm{C}=\mathrm{O}$ lone pair into the broader $\pi$ system. The important result of this is that the corresponding transition dipoles for these states are oppositely aligned, as shown in Figure 1. 
Table 1. Centre $\left(E_{\text {exp }}\right)$ and half width half maximum (HWHM) of Gaussian fit to experimental data. Vertical excitation $\left(E_{\text {vert }}\right)$ energies and oscillator strengths (f) to the first ${ }^{1} \pi \pi^{*}$ state for each ion as calculated using SCS-CC2 and TD-DFT. DNC denotes that the TD-DFT calculation did not converge. ${ }^{a}$ SCS-CC2 calculations employed the def2-TZVP basis set for Irg- $\mathrm{M}^{+}$ions and the def2-SVP basis set for Irg- $\mathrm{M}^{2+}$-Irg ions.

\begin{tabular}{|llllll}
$\begin{array}{l}E_{\text {exp }} \\
(\mathrm{eV})\end{array}$ & $\begin{array}{l}\text { Exp. } \\
\text { HWHM } \\
(\mathrm{eV})\end{array}$ & $\begin{array}{l}\mathrm{E}_{\text {vert }} \\
\mathrm{SCS}-\mathrm{CC} 2^{\mathrm{a}} \\
(\mathrm{eV})\end{array}$ & $\begin{array}{l}\text { Oscillator } \\
\text { Strength } \\
\text { SCS-CC2 } \\
(\mathrm{f})\end{array}$ & $\begin{array}{l}\mathrm{E}_{\text {vert }} \\
\text { TD-DFT } \\
(\mathrm{eV})\end{array}$ & $\begin{array}{l}\text { Oscillator } \\
\text { Strength } \\
\text { TD-DFT } \\
(\mathrm{f})\end{array}$ \\
\hline 3.42 & 0.07 & 3.73 & 0.76 & 4.11 & 0.66 \\
3.97 & 0.24 & 4.08 & 0.63 & 4.36 & 0.56 \\
3.96 & 0.14 & 4.21 & 0.60 & 4.47 & 0.53 \\
4.08 & 0.17 & 4.35 & 0.59 & 4.55 & 0.52 \\
3.36 & 0.11 & 3.58 & 1.52 & 3.90 & 1.34 \\
3.23 & 0.05 & 3.58 & 1.45 & 3.86 & 1.24 \\
3.38 & 0.08 & 3.63 & 1.46 & DNC & DNC
\end{tabular}

As described by other groups, the transition-dipole axis can be thought of as a reaction-axis for OEF control over chemical reactivity. ${ }^{1-3}$ As such, if an electric field was oriented from a positive point-charge at the position as $\mathrm{M}^{\mathrm{Q}^{+}}$, near the ketone and $\mathrm{OH}$ groups as shown in both cases in Figure 2, then the electric field arising from the cation will stabilize (red-shift) the ${ }^{1} \pi \pi^{*}$ state and destabilize (blue-shift) the ${ }^{1} n \pi^{*}$ state. That is, the field from the cation works with the $\pi \rightarrow \pi^{*}$ transition but works against the $\mathrm{n} \rightarrow \pi^{*}$ transition. ${ }^{9-11}$ Ultimately, the stabilization of the $\pi \pi^{*}$ state is in large part due to its polarizability, which leads to significant stabilization despite its almost negligible dipole in the absence of the cation. ${ }^{9-11}$

Experimentally, it is known that the absorption maximum of neutral Irg in acetonitrile is $\lambda_{\max }=273 \mathrm{~nm}(4.54 \mathrm{eV}),{ }^{42,}, 43$ which corresponds to the $\pi \rightarrow \pi^{*}$ transition. The gas-phase $\lambda_{\max }$ for neutral Irg is not reported, so in the absence of this value the experimental red shifts (Figure 3 ) are reported here as shifts relative to this reported acetonitrile $\lambda_{\max }$ value. The experimental shifts, assigned as transitions to the ${ }^{1} \pi \pi^{*}$ state of each system, are listed in Table 2 along with the TD-DFT calculated shift. Figure S8 shows the distances between the center of mass (COM) of the chromophore and the charged atom and are listed in Table 2. From the results, the mono-cations give rise to red shifts up to $1.18 \mathrm{eV}$ measured for $\mathrm{Irg}-\mathrm{H}^{+}$. It is apparent that as the effective radius of mono-cationic $\mathrm{M}^{+}$atom increases, its binding distance from the chromophore increases (see Figure S8A and Table 2). For the di-cationic Irg- $\mathrm{M}^{2+}$-Irg systems shifts are over $1 \mathrm{eV}$, relative to neutral Irg are measured. Shifting of electronic ${ }^{19,20}$ and vibrational ${ }^{57}$ transitions due to bound $\mathrm{M}^{\mathrm{Q}+}$ cations has been reported in gas-phase action spectra by other groups. Electrostatic shifts of electronic states have also been induced using metal chlorides in solution. ${ }^{11}$ Overall, the $\operatorname{Irg}-\mathrm{M}^{\mathrm{Q}^{+}}$action spectra reveal that the amount of red-shift for the ${ }^{1} \pi \pi^{*}$ transition increases as the charge increases from +1 to +2 . Also, the trend is for the redshift to increase as the $\mathrm{M}^{\mathrm{Q}+}$ positions closer to the chromophore.

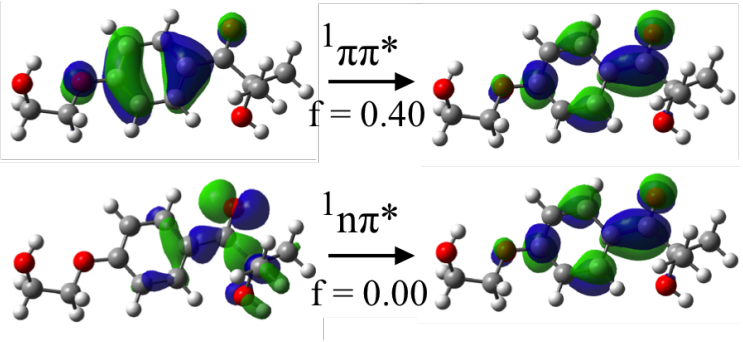

Figure 4. Dominant orbital transitions for the first $\pi \rightarrow \pi^{*}$ transition and $\mathrm{n} \rightarrow \pi^{*}$ transition of neutral Irgacure 2959 (M062X/def2-TZVP) with predicted oscillator strengths (f).

Figure 5A shows a plot of the experimental red shift for the transition to the ${ }^{1} \pi \pi^{*}$ state compared to the calculated TDDFT red-shift, where there is a good correlation. These calculations thus provide good predictions for the shift of the absorption transitions. To assess if the $\mathrm{M}^{\mathrm{Q}+}$ cations are acting effectively as point charges - and hence if the underlying cause of the shift is primarily electrostatic ${ }^{33}$ - TD DFT excitation energies were recalculated with the geometry fixed but with $\mathrm{M}^{\mathrm{Q}+}$ atoms replaced with point charges $\mathrm{P}^{\mathrm{Q}}(\mathrm{Q}=+1 \mathrm{e}$ or $\mathrm{Q}$ $=+2 \mathrm{e})$. Figure $5 \mathrm{~B}$ shows the calculated ${ }^{1} \pi \pi^{*}$ shifts plotted against the shifts calculated for point charges. The monocationic shifts are nearly identically to $\mathrm{P}^{1+}$ point charge values. The di-cationic systems, on the other hand, have a reduced red shift compared to the $\mathrm{P}^{2+}$ point-charge values-falling off the linear trend. This may be due to some bonding interaction between the cation and the Irg molecules, thus reducing the effective charge of the $\mathrm{M}^{2+}$ cation. For the Irg- $\mathrm{M}^{+}$clusters, the correlation between the shift and the point charge values with the $\mathrm{M}^{\mathrm{Q}}$ cations supports the notion that the shifts are an electrostatic effect. ${ }^{33}$ 
Table 2: Calculated distances between chromophore center of mass (COM) and charged atom, electric field strength (V/M) calculated using Coulomb's law, transition energy $\left(E_{\text {exp }}\right)$ shift determined form the PD action spectrum, TD-DFT vertical transition energy shifts $\left(E_{\text {vert }}\right)$ and $\mathbf{E}_{\text {vert }}$ calculated by replacing $\mathbf{M}^{\mathbf{Q}^{+}}$with a point charge.

\begin{tabular}{|c|c|c|c|c|c|}
\hline & $\begin{array}{l}\text { Cation-Irg } \\
\text { Distance } \\
(\AA)\end{array}$ & $\begin{array}{l}\text { Electric Field Strength } \\
(\mathrm{V} / \mathrm{m}) \\
\mathrm{x} 10^{9}\end{array}$ & $E_{\exp }$ Shift & $\begin{array}{l}\text { Evert }_{\text {vift of }} \\
\text { Ion } \\
\text { (TD-DFT) }\end{array}$ & $\begin{array}{l}\text { Evert Shift with }_{\text {voint Charge }} \\
\text { (TD-DFT) }\end{array}$ \\
\hline $\operatorname{Irg}-H^{+}$ & 4.07 & 8.69 & -1.12 & -0.990 & -0.982 \\
\hline $\operatorname{Irg}-\mathrm{Li}^{+}$ & 4.95 & 5.89 & -0.57 & -0.738 & -0.743 \\
\hline $\mathrm{Irg}-\mathrm{Na}^{+}$ & 5.33 & 5.07 & -0.58 & -0.629 & -0.621 \\
\hline $\operatorname{Irg}-K^{+}$ & 5.71 & 4.42 & -0.46 & -0.549 & -0.510 \\
\hline Irg- $\mathrm{Mg}^{2+}-\operatorname{Irg}$ & 5.07 & 11.2 & -1.19 & -1.206 & -1.343 \\
\hline $\operatorname{Irg}-\mathrm{Zn}^{2+}-\operatorname{Irg}$ & 5.07 & 11.2 & -1.31 & -1.238 & -1.364 \\
\hline $\mathrm{Irg}-\mathrm{Ca}^{2+}-\mathrm{Irg}$ & 5.41 & 9.86 & -1.16 & DNC & -1.336 \\
\hline
\end{tabular}

charge $\mathrm{Q}_{\mathrm{M}}($ either $+1 e$ or $+2 e$ ). Values for each system are
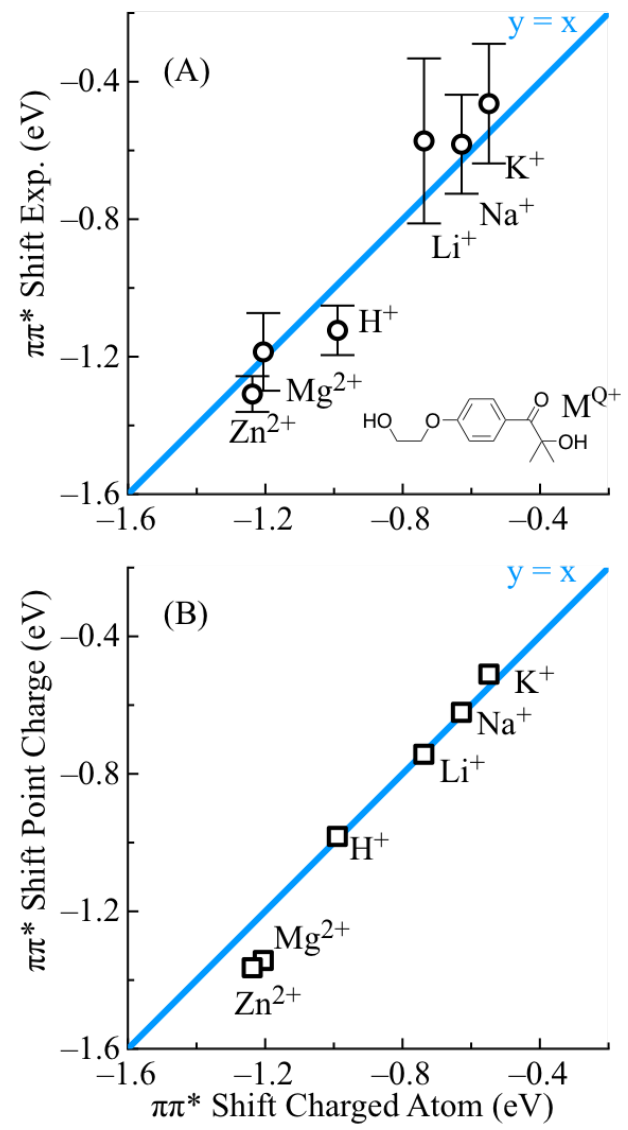

shown in Table 2. Figure 6A shows the plot of the experimental redshift ( $\mathrm{E}_{\exp }$ shift) of the $\pi \rightarrow \pi^{*}$ transition for each ion complex (relative to neutral Irg) against the electric field strength $(E)$ calculated to arise from $\mathrm{M}^{\mathrm{Q}^{+}}$(with error bars from the HWHM from the experimental band, vide supra). A fitted linear function (black line), shows that the experimental band shift of the transition correlates with the electric field strength at the chromophore $\operatorname{COM}\left(R^{2}=0.95\right.$ and y-intercept of $-0.06 \pm$ $0.3 \mathrm{eV}$ ). Importantly, the fit passes through origin in accord with $0 \mathrm{~V} / \mathrm{m}$ electric field affecting zero red shift. In this case of Irg- $\mathrm{M}^{\mathrm{Q}}$ ions, and for other Norrish type-I photoinitiators, ${ }^{9}$

10 the charge-induced dipole alignment-moderated by the polarizability - provides a clear rationalization of the excitedstate energy shift.

For the calculations, the vertical excitation energies to the ${ }^{1} \pi \pi^{*}$ state of Irg- $\mathrm{M}^{+}$and Irg- $\mathrm{M}^{2+}$-Irg ions relative to neutral Irg values are plotted in Figure 6B (triangles). The green trace is the vertical ${ }^{1} \pi \pi^{*}$ excitation energy for neutral Irg in the presence of an increasing static electric field (oriented along the axis of neutral Irg as shown in Figure S1 of Supporting Information). It was found that at higher electric field values, the ${ }^{1} \pi \pi^{*}$ state of interest interacted with a higher lying ${ }^{1} \pi \sigma^{*}$ state (shown as red trace) and this state is dramatically stabilized by the electric field. Although these states interact well-above the $E$ field values for the mono cations, they become mixed around $9 \times 10^{9} \mathrm{~V} / \mathrm{m}$ and invert at electric field strengths above $10 \times 10^{9} \mathrm{~V} / \mathrm{m}$ (These ${ }^{1} \pi \pi^{*}$ and ${ }^{1} \pi \sigma^{*}$ orbitals are shown in Figure S9 of Supporting Information). State-mixing and orbitalmixing is a common feature of molecular species in OEFs. ${ }^{3}$ It is not suspected that the ${ }^{1} \pi \sigma^{*}$ state is involved in the dissociation of the high-field (or any) complexes here. As mentioned above the dissociation products for the $\operatorname{Irg}-\mathrm{Na}^{+}$and $\mathrm{Irg}-\mathrm{K}^{+}$ complexes cleanly photodissociate to $\alpha$-cleavage pathway products, Irg- $\mathrm{Li}^{+}$does in small yields, but the Irg- $\mathrm{H}^{+}$complex and the Irg- $\mathrm{M}^{2+}$-Irg systems do not. The $\alpha$-pathway mechanisms are discussed in the next section.

To further explore the underlying cause of the band shiftand the influence of the metal cation-the electric field strength $(E)$ is calculated using Coulomb's law ${ }^{44}$ (as described in Section SI.6 of Supporting Information). We note that this is a somewhat simplified approximation of the electric field strength, as it is simply the value of the electric field strength at a single distance to a point on the Irg chromophore and the 

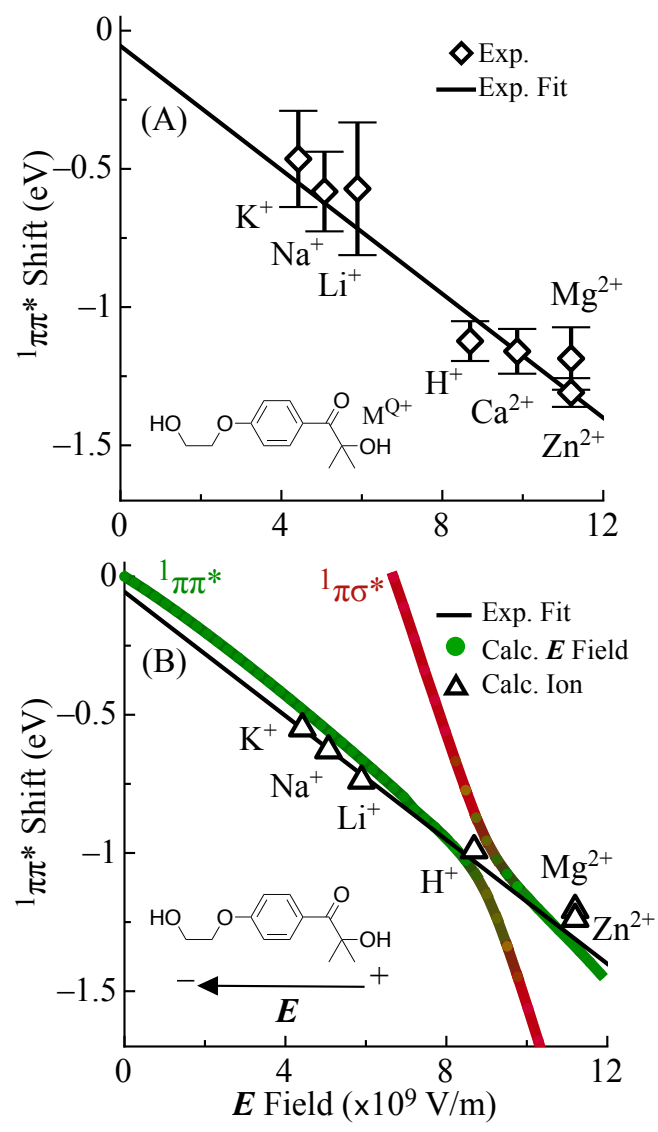

Figure 6. Energy shift of the transition to the first ${ }^{1} \pi \pi^{*}$ state for Irg- $\mathrm{M}^{\mathrm{Q}^{+}}$as a function of the electric field strength $\boldsymbol{E}$. (A) Diamonds are the experimental peak position; error bars are the \pm HWHM of the fitted Gaussian. Black line is a linear regression fit to experimental data. (B) Triangles are the vertical excitation energies of the transition to the ${ }^{1} \pi \pi^{*}$ state (M06-2X/def2-TZVP). The green trace is the ${ }^{1} \pi \pi^{*}$ transition energy (M06-2X/def2TZVP) under static electric fields of different strengths and the red trace follows a ${ }^{1} \pi \sigma^{*}$ state which interacts with the ${ }^{1} \pi \pi^{*}$ state at higher $\boldsymbol{E}$ values (see text).

\section{Photodissociation Pathways}

We have shown that the proximity of the cation affects the amount of red-shift for the key absorbing transition to the ${ }^{1} \pi \pi^{*}$ state of Irg- $\mathrm{M}^{\mathrm{Q}+}$ ions. This achieves the goal of decreasing the photon energy required for excitation. The next required step is efficient formation of radical products via $\alpha$-cleavage. Two pathways resulting in $\alpha$-cleavage are shown in Figure 7 , these are canonical acetophenone-type pathway ${ }^{9,10}$ (Figure 7A) and the more direct ISC pathway (Figure 7B). It is theorized that the efficiency of $\alpha$-cleavage is enhanced when the canonical acetophenone-type photo-dissociation pathway $\left({ }^{1} \pi \pi^{*} \rightarrow{ }^{1} \mathrm{n} \pi^{*}\right.$ $\rightarrow{ }^{3} \pi \pi^{*} \rightarrow{ }^{3} \mathrm{n} \pi^{*} \rightarrow \alpha$-cleavage) is negated by lowering the ${ }^{1} \pi \pi^{*}$ state below the ${ }^{1} \mathrm{n} \pi *$ state. ${ }^{10,11}$ Once achieved, the $\mathrm{S}_{1}$ state is ${ }^{1} \pi \pi^{*}$, and this opens up the more direct ${ }^{1} \pi \pi^{*} \rightarrow{ }^{3} n \pi^{*}$ $\rightarrow \alpha$-cleavage pathway. ${ }^{9-11}$ This direct ISC from the $\mathrm{S}_{1}\left({ }^{1} \pi \pi^{*}\right)$ to the $T_{n}\left({ }^{3} n \pi^{*}\right)$ state must still compete with radiative decay and other non-radiative decay pathways but overall enhancement of ISC is afforded by the decreasing energy gap between the $\mathrm{S}_{1}{ }^{1} \pi \pi^{*}$ state and the $\mathrm{T}_{\mathrm{n}}{ }^{3} \mathrm{n} \pi^{*}$ state. ${ }^{10}$ Dissociation from
${ }^{3} \pi \pi^{*}$ states is predicted to be slow ${ }^{11,58}$ while ISC to the triplet manifold and $\alpha$-cleavage from the ${ }^{3} n \pi^{*}$ state is ultra-fast. ${ }^{43}$
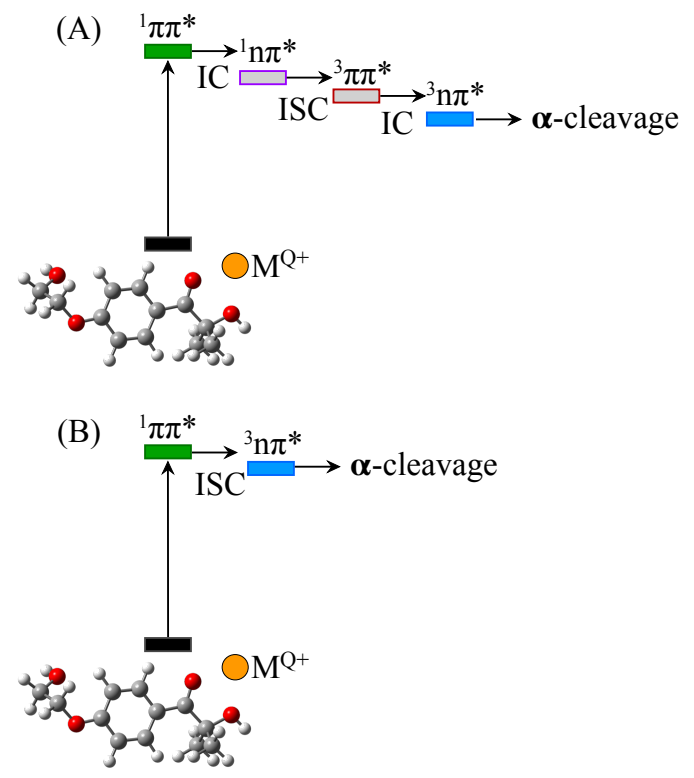

Figure 7. Two $\alpha$-cleavage pathways where (A) the $S_{1}$ state is ${ }^{1} \mathrm{n} \pi *$ character and follows and multistep mechanism and the simpler case (B) where the $S_{1}$ state is ${ }^{1} \pi \pi^{*}$ character pathway.

Using the framework shown in Figure 7, Figure 8 shows the vertical transition energies to the first ${ }^{1} \pi \pi^{*},{ }^{1} \mathrm{n} \pi^{*},{ }^{3} \pi \pi^{*}$ and ${ }^{3} \mathrm{n} \pi^{*}$ states of each $\operatorname{Irg}-\mathrm{M}^{\mathrm{Q}^{+}}$. The Irg- $\mathrm{M}^{\mathrm{Q}+}$-Irg complexes are excluded from this analysis because of their more complex dissociation mass spectra, which presumably arise from other mechanisms. To accurately characterize different spin states, the SCS-CC2 method was used to calculate vertical excitation of these various spin state. CC2 is a robust method for calculating the excitation energies of medium sized molecules and the SCS correction improves results for open shell systems such as the triplet states investigated here. ${ }^{59}$ Calculated ${ }^{1} \pi \pi *$ energies (green rectangles in Figure 8) are compared with experimental energies of the first ${ }^{1} \pi \pi^{*}$ state (black diamonds in Figure 8) and agree well. Therefore, SCS-CC2 better reproduces absolute vertical transition energies, whereas TD-DFT performed well for relative energy shifts but consistently overestimated absolute transition energies by $\sim 0.5 \mathrm{eV}$.

The encroachment of the $\mathrm{M}^{+}$cation to the chromophore (from $\mathrm{Irg}-\mathrm{K}^{+}$to $\mathrm{Irg}-\mathrm{H}^{+}$) red shifts the transition to the $\pi \pi^{*}$ states (green and red rectangles in Figure 8) while raising the transition energy to the $n \pi^{*}$ states (blue and purple rectangles in Figure 8). It is worth noting that in the case of $\mathrm{Irg}-\mathrm{H}^{+}$the $\mathrm{n} \pi *$ state is affected differently because the proton interacts with the $\mathrm{n}$ orbital in a way that cannot be described solely by electrostatic effects. For all Irg- $\mathrm{M}^{\mathrm{Q}+}$ ions, the OEF arising from the presence of the cation stabilizes the ${ }^{1} \pi \pi^{*}$ state such that it shifts below the energy of the ${ }^{1} \mathrm{n} \pi^{*}$ state thus it favors the ISC pathway from $S_{1}\left({ }^{1} \pi \pi^{*}\right)$ to the $T_{n}\left({ }^{3} n \pi^{*}\right)$ state (in accordance with the El Sayed rules). Because ISC can occur from the ${ }^{1} \pi \pi^{*}$ state to the dissociative ${ }^{3} n \pi^{*}$ state, the more 
direct ${ }^{1} \pi \pi^{*} \rightarrow{ }^{3} \mathrm{n} \pi^{*} \rightarrow \alpha$-cleavage pathway (Figure $7 \mathrm{~B}$ ) can dominate.

In order to maximize $\alpha$-cleavage yield, one can drive ISC to the desired dissociative ${ }^{3} n \pi^{*}$ state by minimizing the energy gap between ${ }^{1} \pi \pi^{*}$ and ${ }^{3} n \pi^{*}$ states. This gap is calculated using SCS-CC2/def2-TZVP. The gas-phase PD experiments follow the formation of photoproduct ions and, as such, the PD yield of $\alpha$-cleavage product ions can be reported as a fraction of the total product yield and also as a fraction of the total precursor ion depletion.

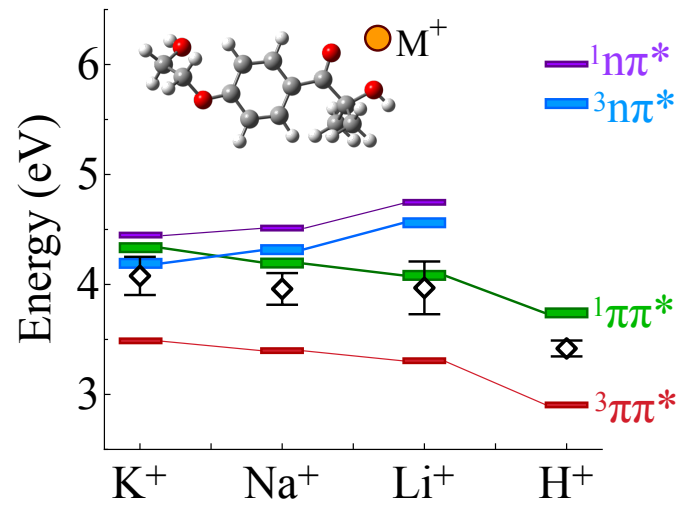

Figure 8. Vertical transition energies to the first ${ }^{1} \pi \pi^{*}$ state (green rectangles), the first ${ }^{3} n \pi^{*}$ state (blue rectangles), the first ${ }^{3} \pi \pi^{*}$ state (red rectangles) and the first ${ }^{3} \mathrm{n} \pi^{*}$ state (purple rectangles) calculated using SCS-CC2/def2-TZVP. Values are compared to the lowest energy feature observed in the action spectra (black diamonds).

Table 3 shows the experimental ratio between the $\alpha$ cleavage photoproduct ion yield $\phi_{\alpha \text {-cleavage }}$ and total PD photoproduct yield $\phi_{\text {products }}$ (or total depletion of the precursor ion $\left.\phi_{\text {depletion}}\right)$ for each $\operatorname{Irg}-\mathrm{M}^{\mathrm{Q}+}$ ion plotted against the energy gap between the first ${ }^{1} \pi \pi^{*}$ and ${ }^{3} \mathrm{n} \pi^{*}$ states as calculated (SCSCC2). The Irg- $\mathrm{Na}^{+}$and $\mathrm{Irg}-\mathrm{K}^{+}$ions have the smallest energy gap between the first ${ }^{1} \pi \pi^{*}$ and ${ }^{3} \mathrm{n} \pi^{*}$ states and dissociate with a yield of $\phi_{\alpha \text {-cleavage }} / \phi_{\text {products }}=0.86\left(\operatorname{Irg}-\mathrm{Na}^{+}\right)$and $\phi_{\alpha \text {-cleavage }} /$ $\phi_{\text {products }}=0.97\left(\mathrm{Irg}-\mathrm{K}^{+}\right)$. The higher $\alpha$-cleavage yield of Irg- $\mathrm{K}^{+}$ can be rationalized as the ${ }^{3} n \pi^{*}$ state being slightly lower in energy than ${ }^{1} \pi \pi^{*}$ state; the corresponding energy difference for Irg- $\mathrm{Na}^{+}$value is slightly positive but within the uncertainty of these values. With an unfavorably large energy gap of 0.5 $\mathrm{eV}$, the Irg- $\mathrm{Li}^{+}$complex dissociated with a small yield of $\phi_{\alpha-}$ cleavage $/ \phi_{\text {products }}=0.0008$. Therefore, under low pressure conditions in the gas-phase, other dissociation pathways can dominate for the Irg- $\mathrm{Li}^{+}$system.

The ratios of $\phi_{\alpha \text {-cleavage }} / \phi_{\text {depletion }}$ are lower than for $\phi_{\alpha \text {-cleavage }} /$ $\phi$ products because we suspect photoproducts are formed below the $m / z$ threshold for the experiment. It is quite possible that $\alpha$ cleavage resulting in ${ }^{\circ} \mathrm{C}_{9} \mathrm{H}_{9} \mathrm{O}_{3}+{ }^{\circ} \mathrm{C}_{3} \mathrm{H}_{6} \mathrm{OH}+\mathrm{M}^{\mathrm{Q}+}$ is occurring and this would go undetected (and not contribute to the $\phi_{\alpha-}$ cleavage value). If this channel was taken into account, then the $\phi_{\alpha \text {-cleavage }} / \phi_{\text {depletion }}$ ratio would be expected to more closely match the $\phi_{\alpha \text {-cleavage }} / \phi_{\text {products }}$ value. Nevertheless, the $\phi_{\alpha-}$ cleavage $/ \phi_{\text {depletion }}$ values follow a similar trend to the $\phi_{\alpha-}$ cleavage $/ \phi_{\text {products }}$ values. Irg- $\mathrm{K}^{+}$has a yield of $\phi_{\alpha \text {-cleavage }} / \phi_{\text {depletion }}=$
0.36 , while Irg- $\mathrm{Li}^{+}$has a yield of $\phi_{\alpha \text {-cleavage }} / \phi_{\text {depletion }}=0.00041$. By tuning the energy gap between ${ }^{1} \pi \pi^{*}$ and ${ }^{3} n \pi^{*}$ quantum states the photochemistry of Irg is altered. This manifests in a yield of $\alpha$-cleavage from $\mathrm{Irg}-\mathrm{K}^{+}$is $\sim 10^{4}$ times larger than for $\operatorname{Irg}-\mathrm{Li}^{+}$

Table 3. Energy gap between the (vertical) ${ }^{1} \pi \pi^{*}$ and ${ }^{3} n \pi *$ states as calculated using SCS-CC2/def2-TZVP. Experimental ratio between photo-dissociation leading to $\alpha$ cleavage $\left(\phi_{\alpha \text {-cleavage }}\right)$ and total observed photo-dissociation yield ( $\left.\phi_{\text {products}}\right)$. Experimental ratio between photodissociation leading to $\alpha$-cleavage $\left(\phi_{\alpha \text {-cleavage }}\right)$ and total observed depletion of the precursor ion yield ( $\left.\phi_{\text {depletion }}\right)$.

\begin{tabular}{lcccc} 
& $\begin{array}{c}\text { SCS-CC2 } \\
\text { Energy Gap } \\
{ }^{3} \mathrm{n} \pi^{*}-1\end{array} \pi^{*}$ & $\begin{array}{c}\text { Exp. } \\
\phi_{\alpha-} \\
\text { cleavage/ } \\
\phi_{\text {products }}\end{array}$ & $\begin{array}{c}\text { Exp. } \\
\phi_{\alpha \text {-cleavagel }}\end{array}$ & $\begin{array}{c}\text { PD } \\
\text { Wepletion } \\
\text { Wavelength } \\
(\mathrm{nm})\end{array}$ \\
\hline $\operatorname{Irg}-\mathrm{K}^{+}$ & -0.15 & 0.97 & 0.36 & 300 \\
$\operatorname{Irg}-\mathrm{Na}^{+}$ & 0.11 & 0.86 & 0.0096 & 296 \\
$\operatorname{Irg}-\mathrm{Li}^{+}$ & 0.48 & 0.0008 & 0.00041 & 310
\end{tabular}

Conclusion:

Using ion trap mass spectrometry, $\mathrm{M}^{\mathrm{Q}^{+}}$cations $\left(\mathrm{H}^{+}, \mathrm{Li}^{+}\right.$, $\mathrm{Na}^{+}, \mathrm{K}^{+}, \mathrm{Zn}^{2+}, \mathrm{Ca}^{2+}, \mathrm{Mg}^{2+}$ ) bound to Irg molecules - either one Irg molecule per $\mathrm{M}^{+}$monocationic atom or two Irg molecules per $\mathrm{M}^{2+}$ dicationic atom - resulting in electric fields of varying strengths that were oriented internally for each Irg molecule. Gas-phase UV PD spectroscopy, combined with TD-DFT calculations, showed that the first ${ }^{1} \pi \pi^{*}$ transition was redshifted proportionally to the strength of the electric field arising from the $\mathrm{M}^{\mathrm{Q}+}$ cation. Red shifts of the transitions to the ${ }^{1} \pi \pi^{*}$ state are observed from the UVC to the UVB or UVA region (depending on the charged atom), in accordance with the predictions by Hill and Coote (2019) that transition energies can be favorably controlled using OEFs.

The radical photoproduct yield of $97 \%$ following $\alpha$ cleavage were measured for Irg- $\mathrm{K}^{+}$, which was the system with the smallest energy gap between the first ${ }^{1} \pi \pi^{*}$ and ${ }^{3} n \pi^{*}$ states (as calculated using SCS-CC2). In contrast, Irg- $\mathrm{Li}^{+}$ions with a ${ }^{1} \pi \pi^{*}$ and ${ }^{3} \mathrm{n} \pi^{*}$ gap of $0.5 \mathrm{eV}$ dissociated with $0.08 \% \alpha$ cleavage. Therefore, with the tuning the ${ }^{1} \pi \pi^{*}-{ }^{3} n \pi^{*}$ energy gap with OEFs, photodissociation can be drastically altered. This study provides combined experimental and theoretical evidence that oriented electric fields can shift photochemical reactivity by tuning the energies of electronic quantum states to affect absorption profiles and photodissociation pathways.

\section{ACKNOWLEDGMENT}

Funding from Australian Research Council grants (AJT: DP200100065 and LP180100550. MLC: CE140100012 and FL170100041) is gratefully acknowledged. This work was also supported by computational resources provided by the Australian Government through the National Computation Infrastructure under the National Computational Merit Allocation Scheme. S.J.P.M., B.I.M., acknowledge support from Australian Government Research Training Program Scholarships 


\section{ASSOCIATED CONTENT}

\section{Supporting Information}

See Supporting Information - as referenced in the text - for experimental details, mass spectra, orientation of static electric field used in calculations, additional structure energies and assignments, additional Gaussian fits for PD action spectra and coordinates for optimized geometries.

The Supporting Information is available free of charge on the ACS Publications website.

\section{AUTHOR INFORMATION}

\section{Corresponding Author \\ *adamt@uow.edu.au (Adam J. Trevitt)}

\section{Notes}

The authors declare no competing financial interests.

1. Ciampi, S.; Darwish, N.; Aitken, H. M.; Díez-Pérez, I.; Coote, M. L., Harnessing electrostatic catalysis in single molecule, electrochemical and chemical systems: a rapidly growing experimental tool box. Chemical Society Reviews 2018, 47 (14), 5146-5164.

2. Shaik, S.; Ramanan, R.; Danovich, D.; Mandal, D., Structure and reactivity/selectivity control by oriented-external electric fields. Chemical Society Reviews 2018, 47 (14), 5125-5145.

3. Stuyver, T.; Danovich, D.; Joy, J.; Shaik, S., External electric field effects on chemical structure and reactivity. Wiley Interdisciplinary Reviews: Computational Molecular Science 2019, e1438.

4. Shaik, S.; Mandal, D.; Ramanan, R., Oriented electric fields as future smart reagents in chemistry. Nature chemistry 2016, 8 (12), 1091

5. Shaik, S.; Danovich, D.; Joy, J.; Wang, Z.; Stuyver, T., Electric-Field Mediated Chemistry: Uncovering and Exploiting the Potential of (Oriented) Electric Fields to Exert Chemical Catalysis and Reaction Control. Journal of the American Chemical Society 2020, 142 (29), 12551-12562.

6. Gorin, C. F.; Beh, E. S.; Kanan, M. W., An Electric FieldInduced Change in the Selectivity of a Metal Oxide-Catalyzed Epoxide Rearrangement. Journal of the American Chemical Society 2012, 134 (1), 186-189.

7. Aragonès, A. C.; Haworth, N. L.; Darwish, N.; Ciampi, S.; Bloomfield, N. J.; Wallace, G. G.; Diez-Perez, I.; Coote, M. L. Electrostatic catalysis of a Diels-Alder reaction. Nature 2016, 531 (7592), 88.

8. Gryn'Ova, G.; Marshall, D. L.; Blanksby, S. J.; Coote, M. L., Switching radical stability by $\mathrm{pH}$-induced orbital conversion. Nature chemistry 2013, 5 (6), 474.

9. Hill, N. S.; Coote, M. L., Internal Oriented Electric Fields as a Strategy for Selectively Modifying Photochemical Reactivity. Journal of the American Chemical Society 2018, 140 (50), 1780017804.

10. Hill, N. S.; Coote, M. L., Strategies for Red-Shifting Type I Photoinitiators: Internal Electric Fields versus Lewis Acids versus Increasing Conjugation. Australian Journal of Chemistry 2019, 72 (8), 627-632.

11. Noble, B. B.; Mater, A. C.; Smith, L. M.; Coote, M. L., The effects of Lewis acid complexation on type I radical photoinitiators and implications for pulsed laser polymerization. Polymer Chemistry 2016, 7 (41), 6400-6412.

12. Menzel, J. P.; Feist, F.; Tuten, B.; Weil, T.; Blinco, J. P.; Barner-Kowollik, C., Light-Controlled Orthogonal Covalent Bond
Formation at Two Different Wavelengths. Angewandte Chemie International Edition 2019, 58 (22), 7470-7474.

13. Wolf, T. J.; Voll, D.; Barner-Kowollik, C.; Unterreiner, A.-N., Elucidating the early steps in photoinitiated radical polymerization via femtosecond pump-probe experiments and DFT calculations. Macromolecules 2012, 45 (5), 2257-2266.

14. Fast, D. E.; Lauer, A.; Menzel, J. P.; Kelterer, A.-M.; Gescheidt, G.; Barner-Kowollik, C., Wavelength-Dependent Photochemistry of Oxime Ester Photoinitiators. Macromolecules 2017, 50 (5), 1815-1823.

15. Blyth, M. T.; Noble, B. B.; Russell, I. C.; Coote, M. L., Oriented Internal Electrostatic Fields Cooperatively Promote Groundand Excited-State Reactivity: A Case Study in Photochemical CO2 Capture. Journal of the American Chemical Society 2019, 142 (1), 606-613.

16. Günther, A.; Nieto, P.; Berden, G.; Oomens, J.; Dopfer, O., IRMPD spectroscopy of metalated flavins: structure and bonding of $\mathrm{M} \mathrm{q}+-$ lumichrome complexes $\left(\mathrm{M} \mathrm{q}+=\mathrm{Li}^{+}+\mathrm{Cs}+, \mathrm{Ag}+, \mathrm{Mg} \mathrm{2+}\right)$. Physical Chemistry Chemical Physics 2014, 16 (27), 14161-14171.

17. Gao, J.; Berden, G.; Rodgers, M.; Oomens, J., Interaction of $\mathrm{Cu}+$ with cytosine and formation of i-motif-like $\mathrm{C}-\mathrm{M}+-\mathrm{C}$ complexes: alkali versus coinage metals. Physical Chemistry Chemical Physics 2016, 18 (10), 7269-7277.

18. Nieto, P.; Günther, A.; Berden, G.; Oomens, J.; Dopfer, O., IRMPD spectroscopy of metalated flavins: structure and bonding of lumiflavin complexes with alkali and coinage metal Ions. The Journal of Physical Chemistry A 2016, 120 (42), 8297-8308.

19. Nieto, P.; Müller, D.; Sheldrick, A.; Günther, A.; Miyazaki, M.; Dopfer, O., Effect of alkali ions on optical properties of flavins: vibronic spectra of cryogenic $\mathrm{M}+$ lumichrome ions $(\mathrm{M}=$ $\mathrm{Li}-\mathrm{Cs})$ in the gas phase. Physical Chemistry Chemical Physics 2018, 20 (34), 22148-22158.

20. Müller, D.; Nieto, P.; Miyazaki, M.; Dopfer, O., Effect of alkali ions on optical properties of flavins: vibronic spectra of cryogenic $\mathrm{M}+$ lumiflavin complexes $(\mathrm{M}=\mathrm{Li}-\mathrm{Cs})$. Faraday discussions 2019, 217, 256-275.

21. Taccone, M. n. I.; Cruz-Ortiz, A. F.; Dezalay, J.; Soorkia S.; Broquier, M.; Grégoire, G.; Sánchez, C. n. G.; Pino, G. A., UV Photofragmentation of Cold Cytosine-M+Complexes $(\mathrm{M}+: \mathrm{Na}+, \mathrm{K}+$, Ag+). The Journal of Physical Chemistry A 2019, 123 (36), 77447750 .

22. Yang, B.; Wu, R.; Polfer, N.; Berden, G.; Oomens, J.; Rodgers, M., IRMPD action spectroscopy of alkali metal cationcytosine complexes: effects of alkali metal cation size on gas phase conformation. Journal of The American Society for Mass Spectrometry 2013, 24 (10), 1523-1533.

23. Marlton, S. J.; McKinnon, B. I.; Ucur, B.; Maccarone, A. T.; Donald, W. A.; Blanksby, S. J.; Trevitt, A. J., Selecting and identifying gas-phase protonation isomers of nicotineH+ using combined laser, ion mobility and mass spectrometry techniques. Faraday discussions 2019, 217, 453-475.

24. Berdakin, M.; Féraud, G.; Dedonder-Lardeux, C.; Jouvet, C.; Pino, G. A., Excited states of protonated DNA/RNA bases. Physical Chemistry Chemical Physics 2014, 16 (22), 10643-10650.

25. Féraud, G.; Esteves-Lopez, N.; Dedonder-Lardeux, C.; Jouvet, C., UV spectroscopy of cold ions as a probe of the protonation site. Physical Chemistry Chemical Physics 2015, 17 (39), 2575525760.

26. Noble, J. A.; Broquier, M.; Gregoire, G.; Soorkia, S.; Pino, G.; Marceca, E.; Dedonder-Lardeux, C.; Jouvet, C., Tautomerism and electronic spectroscopy of protonated 1-and 2aminonaphthalene. Physical Chemistry Chemical Physics 2018, 20 (9), 6134-6145.

27. Matthews, E.; Dessent, C. E., Locating the proton in nicotinamide protomers via low-resolution UV action spectroscopy of electrosprayed solutions. The Journal of Physical Chemistry A 2016, 120 (46), 9209-9216

28. Bull, J. N.; Coughlan, N. J.; Bieske, E. J., ProtomerSpecific Photochemistry Investigated Using Ion Mobility Mass Spectrometry. The Journal of Physical Chemistry A 2017, 121 (32), 6021-6027. 
29. Matthews, E.; Dessent, C. E., Experiment and theory confirm that UV laser photodissociation spectroscopy can distinguish protomers formed via electrospray. Physical Chemistry Chemical Physics 2017, 19 (26), 17434-17440.

30. Matthews, E.; Cercola, R.; Dessent, C., ProtomerDependent Electronic Spectroscopy and Photochemistry of the Model Flavin Chromophore Alloxazine. Molecules 2018, 23 (8), 2036.

31. Marlton, S. J.; McKinnon, B. I.; Ucur, B.; Bezzina, J. P.; Blanksby, S. J.; Trevitt, A. J., Discrimination Between Protonation Isomers of Quinazoline by Ion Mobility and UV-Photodissociation Action Spectroscopy. The Journal of Physical Chemistry Letters 2020.

32. Aragones, A. C.; Haworth, N. L.; Darwish, N.; Ciampi, S.; Bloomfield, N. J.; Wallace, G. G.; Diez-Perez, I.; Coote, M. L., Electrostatic catalysis of a Diels-Alder reaction. Nature 2016, 531 (7592), 88-91.

33. Yue, L.; Li, J.; Zhou, S.; Sun, X.; Schlangen, M.; Shaik, S.; Schwarz, H., Control of product distribution and mechanism by ligation and electric field in the thermal activation of methane. Angewandte Chemie International Edition 2017, 56 (34), 1021910223.

34. Marshall, D. L.; Gryn’ova, G.; Poad, B. L.; Bottle, S. E.; Trevitt, A. J.; Coote, M. L.; Blanksby, S. J., Experimental evidence for long-range stabilizing and destabilizing interactions between charge and radical sites in distonic ions. International Journal of Mass Spectrometry 2019, 435, 195-203.

35. Klinska, M.; Smith, L. M.; Gryn'ova, G.; Banwell, M. G.; Coote, M. L., Experimental demonstration of $\mathrm{pH}$-dependent electrostatic catalysis of radical reactions. Chemical science 2015, 6 (10), 5623-5627.

36. Zhang, L.; Laborda, E.; Darwish, N.; Noble, B. B.; Tyrell, J. H.; Pluczyk, S.; Le Brun, A. P.; Wallace, G. G.; Gonzalez, J.; Coote, M. L., Electrochemical and electrostatic cleavage of alkoxyamines. Journal of the American Chemical Society 2018, 140 (2), 766-774.

37. Noble, B. B.; Smith, L. M.; Coote, M. L., The effect of LiNTf 2 on the propagation rate coefficient of methyl methacrylate. Polymer Chemistry 2014, 5 (17), 4974-4983.

38. Jiang, J. Y.; Smith, L. M.; Tyrell, J. H.; Coote, M. L., Pulsed laser polymerisation studies of methyl methacrylate in the presence of $\mathrm{AlCl} 3$ and $\mathrm{ZnCl} 2$-evidence of propagation catalysis. Polymer Chemistry 2017, 8 (38), 5948-5953.

39. Geng, J.; Li, W.; Zhang, Y.; Thottappillil, N.; Clavadetscher, J.; Lilienkampf, A.; Bradley, M., Radical polymerization inside living cells. Nature chemistry 2019, 11 (6), 578-586.

40. Williams, C. G.; Malik, A. N.; Kim, T. K.; Manson, P. N.; Elisseeff, J. H., Variable cytocompatibility of six cell lines with photoinitiators used for polymerizing hydrogels and cell encapsulation. Biomaterials 2005, 26 (11), 1211-1218.

41. Fedorovich, N. E.; Oudshoorn, M. H.; van Geemen, D.; Hennink, W. E.; Alblas, J.; Dhert, W. J., The effect of photopolymerization on stem cells embedded in hydrogels. Biomaterials 2009, 30 (3), 344-353.

42. Jockusch, S.; Landis, M. S.; Freiermuth, B.; Turro, N. J., Photochemistry and photophysics of $\alpha$-hydroxy ketones. Macromolecules 2001, 34 (6), 1619-1626.

43. Liu, M.; Li, M.-D.; Xue, J.; Phillips, D. L., Time-resolved spectroscopic and density functional theory study of the photochemistry of irgacure-2959 in an aqueous solution. The Journal of Physical Chemistry A 2014, 118 (38), 8701-8707.

44. McNaught, A. D.; Wilkinson, A., IUPAC. Compendium of Chemical Terminology, 2nd ed. (the "Gold Book"). . Blackwell Science Oxford: 1997; Vol. 1669.
45. Segurola, J.; Allen, N.; Edge, M.; Roberts, I., Photochemistry and photoinduced chemical crosslinking activity of acrylated prepolymers by several commercial type I far UV photoinitiators. Polymer degradation and stability 1999, 65 (1), 153160 .

46. Tse, J. R.; Engler, A. J., Preparation of hydrogel substrates with tunable mechanical properties. Current protocols in cell biology 2010, 47 (1), 10.16. 1-10.16. 16.

47. Bryant, S. J.; Nuttelman, C. R.; Anseth, K. S. Cytocompatibility of UV and visible light photoinitiating systems on cultured NIH/3T3 fibroblasts in vitro. Journal of Biomaterials Science, Polymer Edition 2000, 11 (5), 439-457.

48. Wang, Z.; Jin, X.; Dai, R.; Holzman, J. F.; Kim, K., An ultrafast hydrogel photocrosslinking method for direct laser bioprinting. RSC advances 2016, 6 (25), 21099-21104.

49. Xia, H.; Attygalle, A. B., Untrapping Kinetically Trapped Ions: The Role of Water Vapor and Ion-Source Activation Conditions on the Gas-Phase Protomer Ratio of Benzocaine Revealed by IonMobility Mass Spectrometry. Journal of The American Society for Mass Spectrometry 2017, 28 (12), 2580-2587.

50. Klyne, J.; Dopfer, O., Protonation and Sequential Microsolvation of 5-Hydroxyindole: Infrared Photodissociation Spectra of $5 \mathrm{HIH}+-\mathrm{L} \mathrm{n}$ with $\mathrm{L}=\mathrm{Ar}$ and $\mathrm{N} 2(\mathrm{n} \leq 3)$. The Journal of Physical Chemistry B 2018, 122 (47), 10700-10713.

51. Campbell, J. L.; Yang, A. M.-C.; Melo, L. R.; Hopkins, W. S., Studying gas-phase interconversion of tautomers using differential mobility spectrometry. Journal of The American Society for Mass Spectrometry 2016, 27 (7), 1277-1284.

52. Attygalle, A. B.; Xia, H.; Pavlov, J., Influence of Ionization Source Conditions on the Gas-Phase Protomer Distribution of Anilinium and Related Cations. Journal of The American Society for Mass Spectrometry 2017, 28 (8), 1575-1586.

53. Patrick, A. L.; Cismesia, A. P.; Tesler, L. F.; Polfer, N. C., Effects of ESI conditions on kinetic trapping of the solution-phase protonation isomer of p-aminobenzoic acid in the gas phase. International Journal of Mass Spectrometry 2017, 418, 148-155.

54. Kulesza, A. J.; Titov, E.; Daly, S.; Włodarczyk, R.; Megow, J.; Saalfrank, P.; Choi, C. M.; MacAleese, L.; Antoine, R.; Dugourd, P., Excited States of Xanthene Analogues: Photofragmentation and Calculations by $\mathrm{CC} 2$ and Time-Dependent Density Functional Theory. ChemPhysChem 2016, 17 (19), 31293138 .

55. Avila Ferrer, F. J.; Cerezo, J.; Stendardo, E.; Improta, R.; Santoro, F., Insights for an accurate comparison of computational data to experimental absorption and emission spectra: beyond the vertical transition approximation. Journal of chemical theory and computation 2013, 9 (4), 2072-2082.

56. Laurent, A. D.; Jacquemin, D., TD-DFT benchmarks: A review. International Journal of Quantum Chemistry 2013, 113 (17), 2019-2039.

57. Marsh, B. M.; Zhou, J.; Garand, E., Charge transfer in $\mathrm{MOH}(\mathrm{H} 2 \mathrm{O})+(\mathrm{M}=\mathrm{Mn}, \mathrm{Fe}, \mathrm{Co}, \mathrm{Ni}, \mathrm{Cu}, \mathrm{Zn})$ complexes revealed by vibrational spectroscopy of mass-selected ions. Physical Chemistry Chemical Physics 2015, 17 (39), 25786-25792.

58. Ding, L.; Shen, L.; Chen, X.-B.; Fang, W.-H., Solvent effects on photoreactivity of valerophenone: a combined $\mathrm{QM}$ and $\mathrm{MM}$ study. The Journal of organic chemistry 2009, 74 (23), 8956-8962.

59. Brückner, C.; Engels, B., Benchmarking singlet and triplet excitation energies of molecular semiconductors for singlet fission: Tuning the amount of HF exchange and adjusting local correlation to obtain accurate functionals for singlet-triplet gaps. Chemical Physics 2017, 482, 319-338. 
SYNOPSIS TOC: Electrostatic control of excited electronic quantum states are harnessed to tune the photochemistry of a photoinitiator

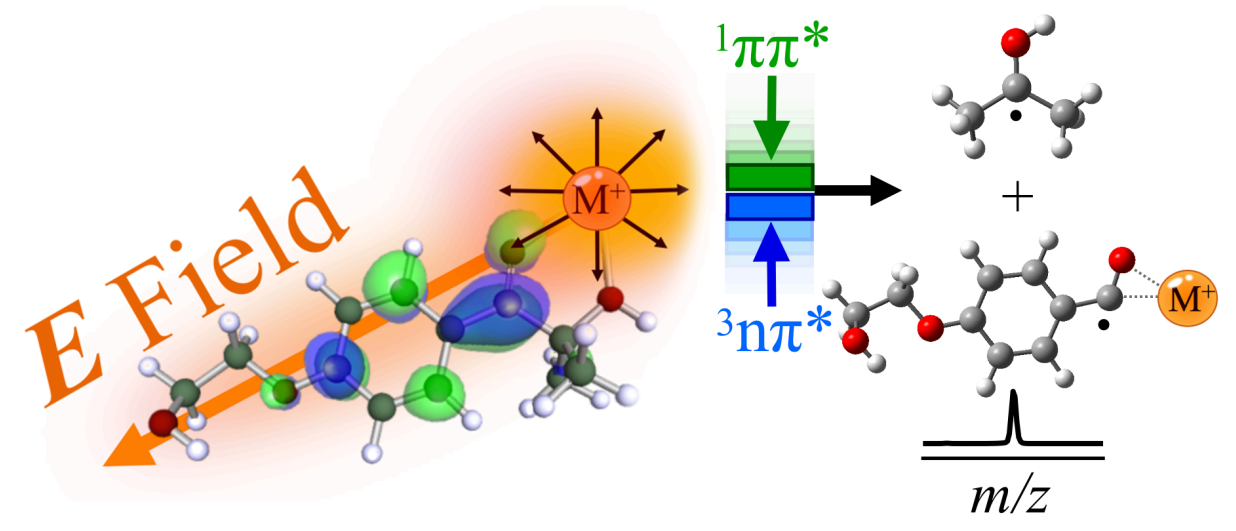

